\title{
New results on the apsidal-motion test to stellar structure and evolution including the effects of dynamic tides
}

\author{
A. Claret $^{1}$ and B. Willems ${ }^{2}$ \\ 1 Instituto de Astrofísica de Andalucía, CSIC, Apartado 3004, 18080 Granada, Spain \\ 2 Department of Physics and Astronomy, The Open University, Walton Hall, Milton Keynes, MK7 6AA, UK \\ e-mail: B.Willems@open.ac.uk
}

Received 21 January 2002 / Accepted 20 March 2002

\begin{abstract}
We revised the current status of the apsidal-motion test to stellar structure and evolution. The observational sample was increased by about $50 \%$ in comparison to previous studies. Classical and relativistic systems were analyzed simultaneously and only systems with accurate absolute dimensions were considered. New interior models incorporating recent opacity tables, stellar rotation, mass loss, and moderate core overshooting were used as theoretical tools to compare the predicted with the observed shifts of the position of the periastron. The stellar models were computed for the precise observed masses and the adopted chemical compositions are consistent with the corresponding tables of opacities to avoid the inherent problems of interpolation in mass and in $(X, Z)$. The derived chemical composition for each individual system was used to infer the primordial helium content as well as a law of enrichment. The values found are in good agreement with those obtained from various independent sources. For the first time, the effects of dynamic tides are taken into account systematically to determine the contribution of the tidal distortion to the predicted apsidal-motion rate. The deviations between the apsidal-motion rates resulting from the classical formula and those determined by taking into account the effects of dynamic tides are presented as a function of the level of synchronism. For systems close to synchronisation, dynamic tides cause deviations with respect to the classical apsidal-motion formula due to the effects of the compressibility of the stellar fluid. For systems with higher rotational angular velocities, additional deviations due to resonances arise when the forcing frequencies of the dynamic tides come into the range of the free oscillation modes of the component stars. The resulting comparison shows a good agreement between the observed and theoretical apsidal-motion rates. No systematic effects in the sense that models are less mass concentrated than real stars and no correlations with the evolutionary status of the systems were detected.
\end{abstract}

Key words. stars: binaries: close - stars: evolution - stars: interiors - stars: oscillations - stars: abundances

\section{Introduction}

It is well known that in close binary systems of stars with eccentric orbits, the tidal and rotational distortions of the components from spherical symmetry lead to secular changes in the position of the periastron. The effect was first investigated by Russell in 1928, and subsequently by Cowling (1938) and Sterne (1939) who found the distortions to depend on the mass distribution of the stars. Comparison of theoretically predicted apsidalmotion rates with the rates derived from observations therefore provides a good tool to test current theories of stellar structure and evolution.

Since the rate of secular apsidal motion has a high dependency on the stellar radii $\left(R^{5}\right)$, only systems with well determined absolute dimensions can be used in the apsidal-motion test. In addition, before any comparison

Send offprint requests to: A. Claret, e-mail: claret@iaa.es between theory and observations can take place, the theoretical stellar models must be able to fit the masses, radii, and effective temperatures of both components at the same age. In the past, these constraints were not always fulfilled, leading to the historical discrepancy where the models were less mass concentrated than real stars (see Claret \& Giménez 1993 for a short review).

Besides the tidal and rotational contributions to the rate of secular apsidal motion, there is also a general relativistic contribution (Levi-Civita 1937). This contribution is often small, but can amount to as much as $50 \%$ and more in binaries with high orbital eccentricities. In the past, these systems were investigated separately in order to avoid that this effect could mask the comparison between theory and observations.

The last systematic comparison between theoretically and observationally determined apsidal-motion rates already dates back to Claret \& Giménez (1993). The main 
conclusion of these authors was that the systematic discrepancies were no longer significantly detected when OPAL opacities, core overshooting and rotation are taken into account, though some systems still showed disagreement. The absolute dimensions of these systems were however not very accurate. About 14 systems with good determination of the absolute dimensions and 10 additional systems with lower quality data were considered in that analysis.

Additional theoretical improvements came from the new treatment of the classical formula for the apsidalmotion rates, which was derived under some simplifications that are not always valid. For instance, the assumption that the star readjusts its shape instantaneously during the revolution around the center of mass of the system is only valid when the orbital period is much larger than the periods of the low-order modes of oscillation. Several authors investigated the importance of resonances and stellar compressibility (Papaloizou \& Pringle 1980; Quataert et al. 1996; and more recently Smeyers \& Willems 2001; Willems \& Claret 2002). Unfortunately, these results were never applied systematically to the apsidal-motion scenario and their contribution could not be quantified.

The availability of new data (there are almost $50 \%$ more systems in our present investigation than in our catalogue of 1993) and the improvement of the stellar models (including opacities and rotation) justify a global revision of the theoretical apsidal-motion rates. We have carried out such a revision taking into account for the first time the effects of stellar compressibility and resonances between dynamic tides and free oscillations modes. Moreover, the stellar models adopted here were computed for the precise observed masses to reduce the internal errors.

\section{The observational data}

Table 1 summarizes the more relevant astrophysical parameters concerning apsidal motion for 21 systems with well determined absolute dimensions and apsidal-motion rates. Searching for appropriate systems is not only a bibliographical survey since it is sometimes hard to separate true good quality data from those presenting only apparent small errors. If one inter-compares the quality of the light curves (mainly $\mathrm{O}-\mathrm{C}$ diagrams) and in some cases their intrinsic complexity, and the input physics of the codes used to analyze them, it can be deduced that the quoted small errors are often rather artificial. Moreover, the use of different instruments, including different photometric systems, surely does not guarantee an equivalent accuracy.

From the spectroscopic point of view, the situation is similar or worse. Radial velocity curves are difficult to obtain: these observations are intrinsically more complex than differential photometry and moreover require larger telescopes for which it is harder to obtain observation time. The ratio between the number of available radial velocity curves and light curves is still very low. Sometimes the masses derived from spectroscopy, together with photometric observations, present no plausible errors. By inspecting the radial velocity curves one can note that for some systems there are only few points and/or the scattering is abnormally high. Clearly, there is no reason why very accurate instruments with accurate techniques should give similar results as those provided by less precise ones. We consider the selected systems shown in Table 1 to be reliable enough for our present purposes ${ }^{1}$.

\section{The physics of the interior models}

The stellar models used in this paper are essentially based on the code described in Claret (1995). The evolution is followed from the ${ }^{12} \mathrm{C}(\mathrm{p}, \gamma){ }^{13} \mathrm{~N}(\beta+\nu){ }^{13} \mathrm{C}(\mathrm{p}, \gamma){ }^{4} \mathrm{~N}$ reaction until the end of the triple- $\alpha$ process. If required, more evolved structures and/or tracks including the Pre MainSequence phase can also be generated.

The range of possible chemical compositions was enlarged and the adopted opacities are those by Iglesias \& Rogers (1996) complemented by the calculations of Alexander \& Ferguson (1994) or Kurucz (1994) for low temperatures. Convection is considered following the classical mixing-length theory (Böhm-Vitense 1958) although alternative theories can also be adopted. The current value of the mixing-length parameter, $\alpha$, is 1.52 which reproduces well the solar properties and most part of the DLEBS (double-lined eclipsing binary systems). The adopted core overshooting parameter was $\alpha_{\mathrm{ov}}=0.20$. Alternative values were also used along the paper to ascertain its effects on the comparison with the observational data.

Of particular interest for our study is how the apsidalmotion constants can be derived from the theoretical stellar models. In order to obtain these constants we integrate the differential equations of Radau

$$
\frac{a \mathrm{~d} \eta_{j}}{\mathrm{~d} a}+\frac{6 \rho(a)}{\bar{\rho}(a)}\left(\eta_{j}+1\right)+\eta_{j}\left(\eta_{j}-1\right)=j(j+1), j=2,3,4
$$

${ }^{1}$ References to Table 1: 1) Burns et al. (1996), 2) Andersen et al. (1987), 3) Andersen et al. (1984), 4) Imbert (1987), 5) Clausen (1991), 6) Popper (1987), 7) Claret et al. (1995), 8) Clausen et al. (1977), 9) Andersen \& Giménez (1985), 10) Giménez \& Quintana (1992), 11) Hill \& Holmgren (1995), 12) Simon et al. (1994), 13) Giménez \& Clausen (1994), 14) Andersen et al. (1983), 15) Giménez et al. (1986), 16) Clausen et al. (1986), 17) Holmgren et al. (1995), 18) Wolf (1995), 19) Barembaum \& Etzel (1995), 20) Clausen (1996), 21) Claret \& Giménez (1993), 22) Caton \& Burns (1993), 23) Torres et al. (2000), 24) Torres et al. (1999), 25) Andersen (1983), 26) Andersen (1991), 27) Lacy \& Frueh (1985), 28) Andersen et al. (1993), 29) Clausen \& Giménez (1991), 30) Popper \& Hill (1991), 31) Andersen et al. (1985), 32) Wolf (2000), 33) Popper (1987), 34) Popper \& Hill (1991), 35) Kämper (1986), 36) Wolf et al. (2001), 37) Andersen \& Clausen (1989), 38) Tomkin (1983). 
Table 1. Astrophysical parameters of EBS showing apsidal motion.

\begin{tabular}{|c|c|c|c|c|c|c|c|c|c|c|c|}
\hline Name & $m_{1}$ & $m_{2}$ & $R_{1}$ & $R_{2}$ & $e$ & $\dot{\omega}_{\text {obs }}$ & $\overline{k_{2 \mathrm{obs}}}$ & $P$ & $V_{\mathrm{r} 1}$ & $V_{\mathrm{r} 2}$ & Ref. \\
\hline BW Aqr & 1.488 & 1.386 & 2.064 & 1.788 & 0.17 & 0.0009 & -2.18 & 6.72 & 13.5 & 9.6 & $4,5,26$ \\
\hline BD-16 6074 & 0.022 & 0.021 & 0.043 & 0.044 & 0.01 & 0.0001 & 0.12 & & & & \\
\hline V539 Ara & 6.24 & 5.31 & 4.50 & 3.42 & 0.053 & 0.021 & -2.27 & 3.17 & 75 & 48 & 20 \\
\hline HD 161783 & 0.07 & 0.06 & 0.08 & 0.08 & 0.001 & 0.002 & 0.08 & & & & \\
\hline EM Car & 22.89 & 21.42 & 9.34 & 8.33 & 0.120 & 0.081 & -2.34 & 3.41 & 151 & 130 & 37 \\
\hline HD 161783 & 0.32 & 0.33 & 0.17 & 0.14 & 0.0005 & 0.010 & 0.09 & & & & \\
\hline QX Car & 9.267 & 8.480 & 4.289 & 4.051 & 0.278 & 0.01222 & -2.15 & 4.48 & 120 & 110 & 14,15 \\
\hline HD 161783 & 0.122 & 0.122 & 0.091 & 0.091 & 0.003 & 0.00022 & 0.05 & & & & \\
\hline PV Cas & 2.827 & 2.768 & 2.243 & 2.287 & 0.0325 & 0.0186 & -2.45 & 1.75 & 65 & 65 & $18,19,33$ \\
\hline HD 161783 & 0.050 & 0.054 & 0.035 & 0.035 & 0.0003 & 0.0004 & 0.05 & & & & \\
\hline CW Cep & 13.52 & 12.08 & 5.685 & 5.177 & 0.0293 & 0.05902 & -2.13 & 2.73 & 132 & 138 & $21,29,30$ \\
\hline HD 161783 & 0.59 & 0.29 & 0.130 & 0.129 & 0.0006 & 0.00080 & 0.06 & & & & \\
\hline EK Cep & 2.029 & 1.124 & 1.579 & 1.315 & 0.109 & 0.00101 & -2.06 & 4.43 & 23 & 10.5 & $6,7,38$ \\
\hline HD 161783 & 0.023 & 0.012 & 0.007 & 0.006 & 0.003 & 0.00015 & 0.13 & & & & \\
\hline V477 Cyg & 1.79 & 1.35 & 1.57 & 1.27 & 0.307 & 0.00661 & -2.24 & 2.35 & 64 & 50 & 10,21 \\
\hline HD 161783 & 0.12 & 0.07 & 0.05 & 0.04 & 0.003 & 0.00018 & 0.09 & & & & \\
\hline V1143 Cyg & 1.391 & 1.347 & 1.346 & 1.323 & 0.540 & 0.00074 & -2.32 & 7.64 & 18 & 28 & 1,2 \\
\hline HD 161783 & 0.016 & 0.013 & 0.023 & 0.023 & 0.003 & 0.00015 & 0.22 & & & & \\
\hline Y Cyg & 17.57 & 17.04 & 5.93 & 5.78 & 0.1458 & 0.0618 & -1.98 & 3.00 & 147 & 138 & $11,12,17$ \\
\hline HD 161783 & 0.27 & 0.26 & 0.07 & 0.07 & 0.0023 & 0.0003 & 0.03 & & & & \\
\hline V364 Lac & 2.333 & 2.296 & 3.307 & 2.985 & 0.2873 & 0.00258 & -2.41 & 7.35 & 45 & 15 & 24 \\
\hline HD 161783 & 0.015 & 0.025 & 0.038 & 0.035 & 0.0014 & 0.00033 & 0.09 & & & & \\
\hline GG Lup & 4.116 & 2.509 & 2.379 & 1.725 & 0.150 & 0.0181 & -2.15 & 1.85 & 89 & 65 & 28 \\
\hline HD 161783 & 0.040 & 0.024 & 0.025 & 0.019 & 0.005 & 0.0006 & 0.18 & & & & \\
\hline U Oph & 5.198 & 4.683 & 3.438 & 3.005 & 0.0031 & 0.0779 & -2.23 & 1.68 & 107 & 87 & $26,34,35$ \\
\hline HD 161783 & 0.113 & 0.090 & 0.044 & 0.055 & 0.0003 & 0.0012 & 0.04 & & & & \\
\hline V451 Oph & 2.776 & 2.356 & 2.640 & 2.028 & 0.0125 & 0.0118 & -2.46 & 2.20 & 41 & 30 & 16,36 \\
\hline HD 161783 & 0.063 & 0.052 & 0.031 & 0.028 & 0.0015 & 0.0007 & 0.06 & & & & \\
\hline GG Ori & 2.342 & 2.338 & 1.852 & 1.830 & 0.2218 & 0.00061 & -2.41 & 6.63 & 20 & 20 & 23 \\
\hline HD 161783 & 0.016 & 0.017 & 0.025 & 0.025 & 0.0022 & 0.00025 & 0.72 & & & & \\
\hline AG Per & 5.36 & 4.90 & 2.99 & 2.60 & 0.0710 & 0.0264 & -2.14 & 2.03 & 95 & 71 & 13 \\
\hline HD 161783 & 0.16 & 0.13 & 0.07 & 0.07 & 0.0010 & 0.0022 & 0.05 & & & & \\
\hline IQ Per & 3.521 & 1.737 & 2.446 & 1.503 & 0.076 & 0.0144 & -2.33 & 1.74 & 68 & 44 & $1,22,26,27$ \\
\hline HD 161783 & 0.067 & 0.031 & 0.026 & 0.017 & 0.0010 & 0.0022 & 0.10 & & & & \\
\hline$\zeta$ Phe & 3.930 & 2.550 & 2.85 & 1.85 & 0.0113 & 0.0373 & -2.22 & 1.67 & 85 & 75 & $20,21,25$ \\
\hline HD 161783 & 0.045 & 0.026 & 0.02 & 0.02 & 0.0020 & 0.0055 & 0.08 & & & & \\
\hline VV Pyx & 2.098 & 2.098 & 2.167 & 2.167 & 0.0956 & 0.00142 & -2.47 & 4.60 & 23 & 23 & 3 \\
\hline HD 161783 & 0.018 & 0.018 & 0.020 & 0.020 & 0.0009 & 0.00045 & 0.23 & & & & \\
\hline V760 Sco & 4.980 & 4.620 & 3.013 & 2.640 & 0.0265 & 0.0438 & -2.19 & 1.73 & 96 & 86 & 31,32 \\
\hline HD 161783 & 0.090 & 0.073 & 0.066 & 0.053 & 0.0010 & 0.0032 & 0.07 & & & & \\
\hline V1647 Sgr & 2.189 & 1.972 & 1.831 & 1.666 & 0.4143 & 0.00546 & -2.38 & 3.28 & 80 & 70 & $8,9,32$ \\
\hline HD 161783 & 0.037 & 0.033 & 0.018 & 0.017 & 0.0005 & 0.00006 & 0.03 & & & & \\
\hline
\end{tabular}

where

$\eta \equiv \frac{a}{\epsilon_{j}} \frac{\mathrm{d} \epsilon_{j}}{\mathrm{~d} a}$

(see for example, Kopal 1959). In these equations, $a$ is the mean radius of the configuration and $\epsilon_{j}$ is a measure of the deviation from sphericity. Integration of the above differential equations then yields the values of the apsidalmotion constants through the simple equation

$k_{j}=\frac{j+1-\eta_{j}(R)}{2\left(j+\eta_{j}(R)\right)}$

where $R$ refers the values of $\eta_{j}$ at the star's surface. Note that the deviations from sphericity associated with the $j$ 's of order higher than 2 do not contribute significantly to the total. As we cannot separate the individual contribution of each star to the observed apsidal motion we must use an appropriate mean value (see Eqs. (4) and (11)).

It is expected that fast rotating stars are more mass concentrated than those presenting low rotational rates. From the theoretical point of view, rotation should therefore affect the mass distribution, and in particular, the apsidal-motion constants. Stothers (1974) was the first to quantify this effect. In 1993, Claret \& Giménez made a first attempt to evaluate the contribution of the rotation and more recently, Claret (1999) introduced a more elaborate method to treat rotation in his code. This method 
is based on the work by Kippenhahn \& Thomas (1970) and takes into account three contributions to the total gravitational potential: the spherical symmetric one, that due to the rotation, and the integral which describes the distortion (Claret 1999, Eq. (1)).

\section{Confrontation observation-theory}

\subsection{Ages and effective temperatures}

As commented before, the apsidal-motion test to stellar structure will only be reliable if the adopted stellar models are capable to reproduce well the basic properties of DLEBS. For a given chemical composition the theoretical isochrone must, for instance, be compatible with the observed masses, radii and effective temperatures of both components simultaneously. One of the weakest points of stellar physics, and in particular, of the study of eclipsing binaries, is that the chemical composition is not known accurately, which increases the number of free parameters usually adopted in stellar evolution codes. On the other hand, given the range of effective temperatures of our observational sample, the importance of the mixing-length parameter $\alpha$ is not very high.

The lack of observational support to determine the chemical composition limits our conclusions and any other sources of errors must be eliminated as much as possible to increase the reliability of our results. In order to check the capability of our stellar models to predict the astrophysical properties of the observational sample, we have computed specific models for the precise observed masses. The adopted chemical compositions also correspond to the respective tables of opacities. No interpolation was used to infer the chemical composition in order to minimize the internal errors due to interpolation processes.

The main parameters used as a guide to check the stellar models were the observed masses and radii which are free from pre-calibrations. Effective temperatures are also a good tool, but unfortunately these astrophysical quantities depend on calibrations and/or atmosphere models. A clear example is the system EK Cep that is a very young system with a secondary which is still in the Pre Main-Sequence phase (Claret et al. 1995). The definition of the effective temperature for this system, which is probably still surrounded by interstellar material, is not a clear question. In spite of this, the effective temperatures were also used to search for the best solution for each system, using the temperature ratio $-T_{\text {eff } 2} / T_{\text {eff } 1}-$ which is a better determined quantity. However, we give more weight to the masses and radii.

Considering the above remarks, we subject our models to the first test concerning the ages of the selected systems. As can be noticed in Fig. 1 the ages of both components of each system are approximately the same, as required. However, for some systems, namely, AG Per, V760 Sco, V1143 Cyg and V477 Cyg this constraint is not satisfactorily fulfilled. These binaries are known to present problems of location in the HR Diagram, mainly

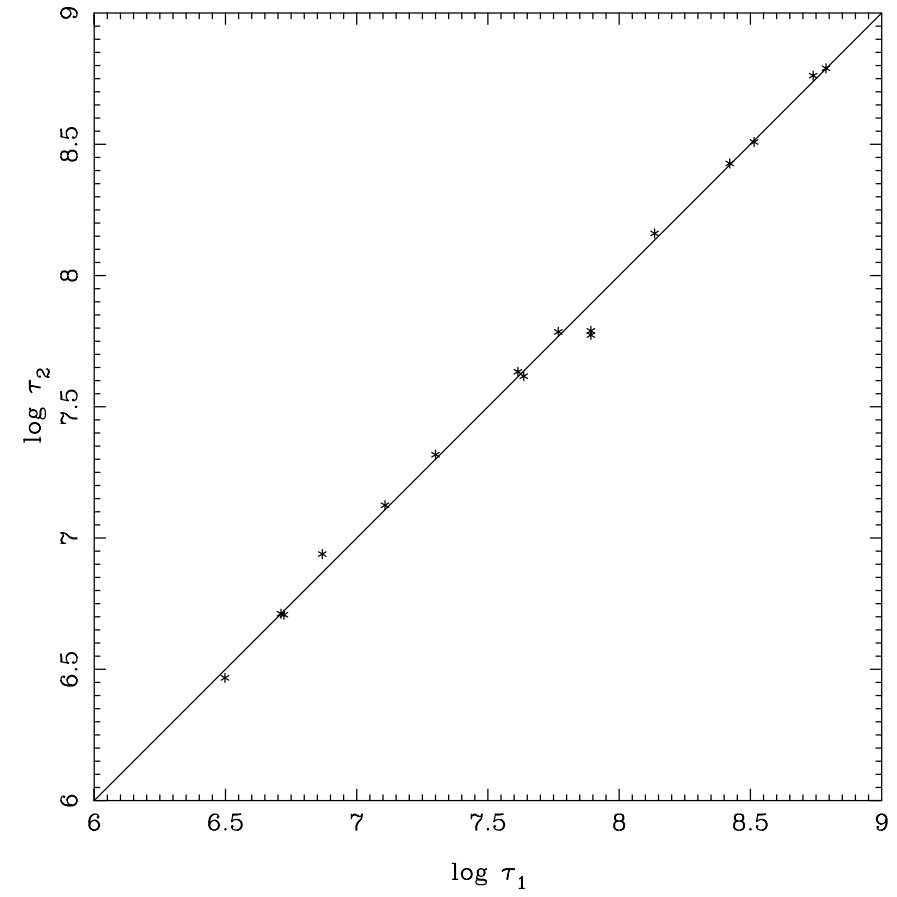

Fig. 1. The inferred ages for the primaries $\tau_{1}$ and secondaries $\tau_{2}$ as given by the best chemical composition fitting.

concerning their ages since they are still relatively young systems. In fact, this is one of the most used arguments to explain such anomalous locations. This may be true only in part though because there are young binaries which do not show this kind of discrepancy. We have carried out some checks changing the chemical composition or including the Pre Main-Sequence phase in the theoretical calculations but the problem was not solved satisfactorily. These binaries are therefore not represented in Figs. 1 and 2. They also do not seem to be the only problematic systems. Clausen et al. (1999), for example, showed that some binaries with masses in the range $0.7-1.0 M_{\odot}$ also pose a problem to the current evolutionary stellar models since the theoretical models for the secondaries predict smaller radii and higher effective temperatures than observed. When we take a more extensive sample of binaries with good determination of absolute dimensions (e.g. that by Andersen 1991) we find a similar behaviour for some systems. This poses a challenge to the evolutionary models. Many aspects of the input physics surely must be revised and improved. In addition, the observational data should also provide more confident and additional information than that which is usually obtained from traditional light and radial velocity curve studies.

Concerning the effective temperatures, the comparison with theoretical predictions shows a good agreement (Fig. 2). As mentioned before, we consider the effective temperatures as a good guide but not a secure parameter to compare with their theoretical counterparts. As explained, we give more weight to the comparison mass$\log g$ because in doing so no pre-calibration is necessary and eventual systematic effects can be reduced. 
A. Claret and B. Willems: New results on the apsidal-motion test to stellar structure

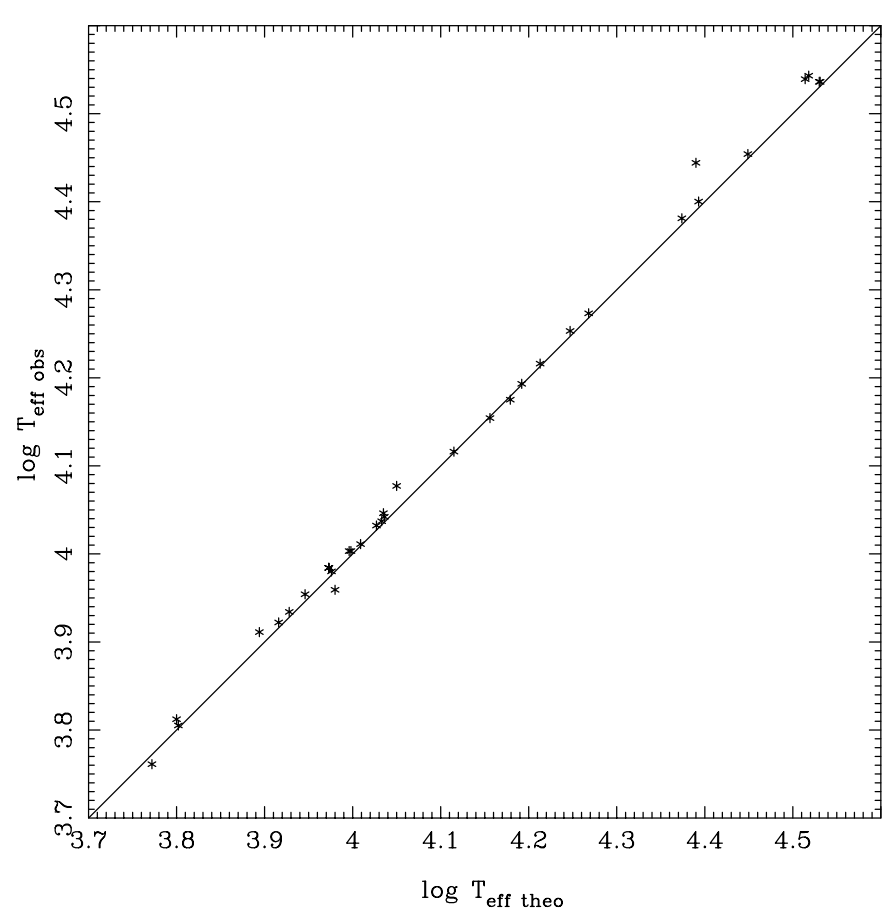

Fig. 2. The theoretical and observed effective temperatures for the primaries and secondaries. Same remarks as in Fig. 1.

\subsection{The primordial helium content and the law of enrichment}

It is possible to investigate the primordial helium content and a "law of metallic enrichment" taking into account the inferred chemical composition of the eclipsing binaries discussed in Sect. 4.1. In our early papers on apsidal motion in DLEBS (Claret \& Giménez 1993; Claret 1997), a single average chemical composition of $(X, Z)=(0.70$, $0.02)$ was adopted, which lead to good general results. To refine the comparison observation-theory, we here investigate each system individually and we consider the values of $(X, Z)$ which gives the best fit in the planes mass-log $g$ and $\log g-\log T_{\text {eff. }}$

In order to increase our sample of DLEBS we have introduced 9 additional systems: GZ Cma, YZ Cas, WX Cep, AI Hya, TZ Men, V1031 Ori (Andersen 1991), HS Hya (Torres et al. 1997), EE Peg (Linnell et al. 1996), AI Phe (Andersen et al. 1988). We selected these binaries due to the fact that there is observational evidence to support the determination of their metallicities. The results are shown in Fig. 3. The derived value of the primordial helium content is $Y_{\mathrm{p}}=0.238 \pm 0.011$. We performed some tests using, for example, the complete sample of DLEBS compiled by Andersen (1991) and found a variation in $Y_{\mathrm{p}}$ around $10 \%$. If we use only the sample of stars showing apsidal motion, the uncertainty in $Y_{\mathrm{p}}$ is also of the same order. We can therefore conclude that, within the mentioned variation, the inferred primordial helium is almost independent of the sample. The influence of the four problematic systems AG Per, V760 Sco, V1143 Cyg, and V477 Cyg mentioned in Sect. 4.1 on the determination of $Y_{\mathrm{p}}$ is not large.

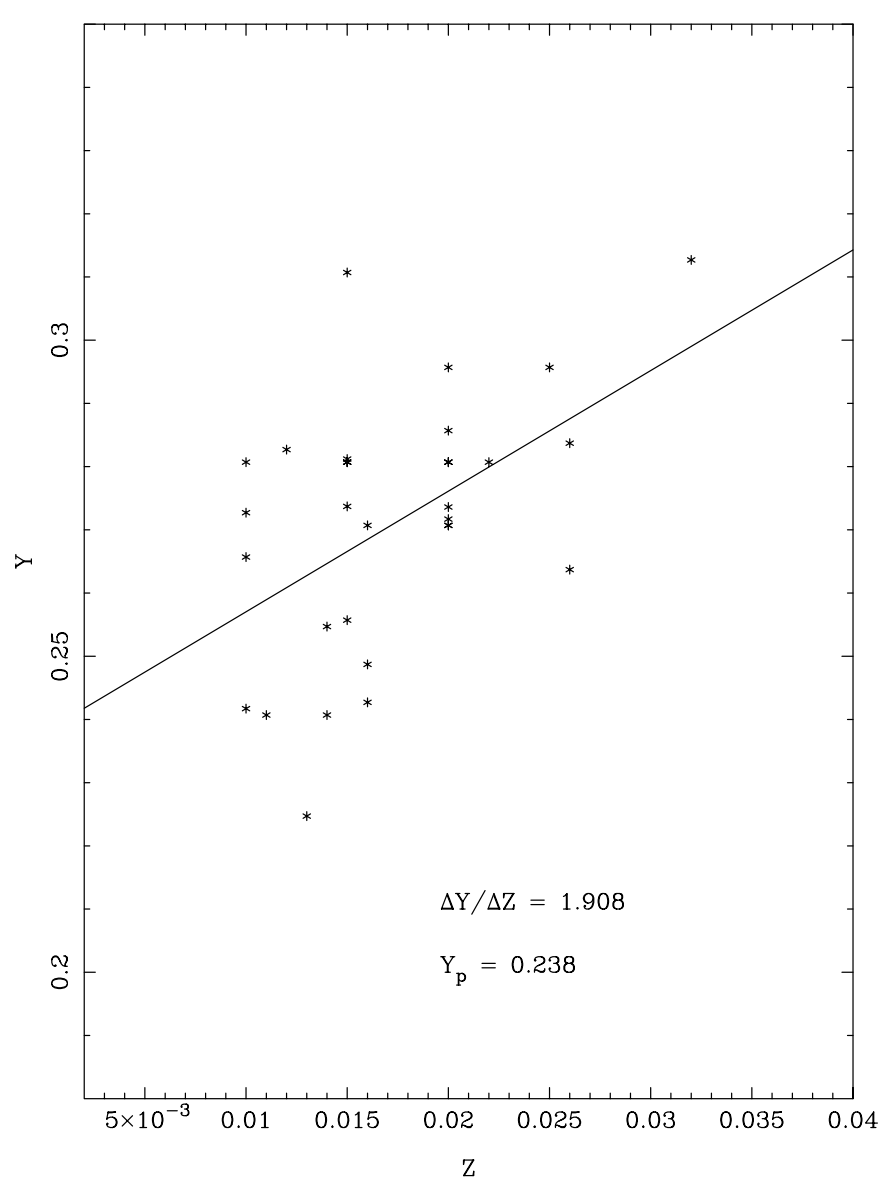

Fig. 3. The primordial helium content and the metallic enrichment law inferred from the fitting of evolutionary models to the absolute dimensions of the DLEBS listed in Table 1 (with nine additional systems with observational evidence for the determination of the metal content).

The same conclusion does not apply to the law of enrichment. The error of \pm 0.608 in $\Delta Y / \Delta Z$ is a first evidence that this parameter is much more sensitive to the selected sample than the primordial helium content. The ratio $\Delta Y / \Delta Z=1.908$ itself also depends strongly on the previous selection process. Several tests were carried out to prove this dependency and indeed, the changes in the ratio may be of the order of $50 \%$ or more. This may have interesting implications as, for example, that the law of enrichment might not be universal. In spite of the above speculation and the numerical tendencies often found in a linear least-squares fitting procedure, the characteristics of the metallic enrichment are, in our opinion, rather local than universal given the contributions to the local enrichment as the low metallic content, mixing phenomena, including mass transfer in close binary systems (see Maeder 1983). In addition, the dependence of $Y$ with $\Delta Y / \Delta Z$ may not be a simple linear approximation since this is only the first term of a Taylor series. The inclusion of systems which are in a very different chemical environment should be considered to test the universality of the derivative. The importance of observing EBS's in different galaxies such as the LMC, the SMC or Andromeda 
is obvious. Another possible implication of the inaccuracy in the determination of $\Delta Y / \Delta Z$ are the systematic errors in the effective temperatures although we believe we have reduced these somewhat in this paper. Possibly the fact that some systems can be represented by more than one chemical composition may also increase the dispersion. Additional processes like rotation surely add more noise to the problem not only due to the classical point that rotating models are cooler and less luminous than their non-rotating counterparts but also due to the transport of angular momentum and rotationally induced mixing.

However, there are more restrictions to the interpretation of the results shown in Fig. 3. Even for systems with a well determined metallicity content, we cannot forget that the hydrogen (or helium) content is also fundamental to determine the structure and evolution of the stars. If the empirical values of the metallicity and hydrogen (or helium) abundance are not known simultaneously, we have to use semi-empirical enrichment laws to compute the theoretical models, which obviously introduces additional uncertainties in the results. The high dispersion found in the semi-empirical $Z-Y$ plane limits the validity of the derived enrichment law. This inconsistency yielded Claret (1995) to compute stellar evolution models for a wide range of chemical compositions. Given the actual situation we should therefore take (and interprete) the above results with caution.

Two final remarks can be made: 1) it should be stressed that the chemical composition is a free parameter and that although the inferred values of $Y_{\mathrm{p}}$ and $\Delta Y / \Delta Z$ are in good agreement with those obtained from independent sources, the real errors are much larger than those presented before considering the uncertainties associated with the interior models. The errors 0.608 and 0.011 refer only to the numerical adjustment. An optimistic estimate of the error is, in our opinion, at least of the order of $15 \%$ for $Y_{\mathrm{p}}$, and much more for $\Delta Y / \Delta Z$. 2) Considering all the difficulties mentioned above, it is encouraging that the inferred values of $Z$ and $\Delta Y / \Delta Z$ obtained here confirm the adopted average chemical composition used previously (Claret \& Giménez 1993; Claret 1997). The present results also confirm, within the theoretical uncertainties, the values found by Ribas et al. (2000) who used the same stellar evolution code.

\section{The apsidal-motion test}

\subsection{The classical and general relativistic contribution}

As commented briefly before, it is not possible to separate the individual contribution of each component to the total apsidal-motion rate. The mean observed apsidal-motion constant taking into account the contribution of both stars can be written as a function of the stellar masses $m_{i}$, the stellar radii $R_{i}$, the eccentricity $e$, the orbital period $P$, and the apsidal-motion period $U$. The function takes the form

$\bar{k}_{2 \mathrm{obs}}=\frac{1}{c_{21}+c_{22}} \frac{P}{U}$

where

$c_{2 i}=\left[\left(\frac{\Omega_{i}}{\Omega_{\mathrm{K}}}\right)^{2}\left(1+\frac{m_{3-i}}{m_{i}}\right) f(e)+\frac{15 m_{3-i}}{m_{i}} g(e)\right]\left(\frac{R_{i}}{A}\right)^{5}$.

In the above equations, $A$ is the semi-major axis of the orbit, and $f$ and $g$ are functions of the orbital eccentricity defined as

$f(e)=\left(1-e^{2}\right)^{-2}$
$g(e)=\frac{\left(8+12 e^{2}+e^{4}\right) f(e)^{2.5}}{8}$

The factor $\Omega_{i} / \Omega_{\mathrm{K}}$ in the first term between the square brackets in Eq. (5) is the ratio between the rotational angular velocity of component $i$ and the average orbital angular velocity. The relation between the apsidal-motion period $U$ and the observed rate of apsidal motion has a very simple form

$U=\frac{360 P}{\dot{\omega}_{\mathrm{obs}}}$

The relativistic correction to the advance of the periastron does not depend on the stellar structure and is governed solely by the orbital elements and stellar masses

$\frac{P}{U^{\prime}}=6.35 \times 10^{-6} \frac{\left(m_{1}+m_{2}\right)}{A\left(1-e^{2}\right)}$,

where $U^{\prime}$ is the associated period of apsidal-motion. As an alternative to the theory of General Relativity, Moffat (1984, 1989) proposed a gravitational theory based on a non-symmetric tensor and a non-symmetrical affine connection which predicted slower shifts of the position of the periastron. However, this possibility brought more problems than answers. Moreover, the predictions made using the General Theory of Relativity are in good agreement with observational data (see Claret 1997 for a detailed discussion). Therefore, in this paper we adopted the GR equation to correct the observed advance of the position of the periastron.

The resulting apsidal-motion rate to be compared with theory is then

$\dot{\omega}_{\text {clas }}=\dot{\omega}_{\mathrm{obs}}-\dot{\omega}_{\mathrm{GR}}$.

This equation, combined with Eqs. (4) and (8) will allow us to compare directly the observationally determined apsidal-motion constants with those derived from stellar evolution models by means of the equation

$\bar{k}_{2 \text { theo }}=\frac{c_{21} k_{21 \text { theo }}+c_{22} k_{22 \text { theo }}}{c_{21}+c_{22}}$.

The effects of dynamic tides on the apsidal-motion shift cannot be written in a closed form. Instead, these effects 
must be computed for each case individually. In the next section we discuss the main aspects and approximations used to derive the equations governing the rate of change of the longitude of the periastron within the framework of the theory of dynamic tides.

\subsection{The influence of dynamic tides}

In close binary systems of stars with non-synchronously rotating components, each star is subject to the dynamic tides raised by its companion. The most commonly used approach to study the time-dependent tidal action exists in expanding the tide-generating potential $\varepsilon_{\mathrm{T}} W(\boldsymbol{r}, t)$ in a Fourier series in terms of the companion's mean Keplerian motion $\Omega_{\mathrm{K}}$ (e.g., Zahn 1970; Polfliet \& Smeyers 1990). If $m_{1}$ is assumed to be uniformly rotating and if $m_{2}$ is considered to be a point mass, the expansion takes the form

$$
\begin{gathered}
\varepsilon_{\mathrm{T}} W(\boldsymbol{r}, t)=-\varepsilon_{\mathrm{T}} \frac{G m_{1}}{R_{1}} \sum_{\ell=2}^{4} \sum_{m=-\ell}^{\ell} \sum_{k=-\infty}^{\infty} c_{\ell, m, k}\left(\frac{r}{R_{1}}\right)^{\ell} \\
\times Y_{\ell}^{m}(\theta, \phi) \exp \left(-\mathrm{i} k \Omega_{\mathrm{K}} \tau\right) \exp \left[\mathrm{i}\left(k \Omega_{\mathrm{K}}+m \Omega_{1}\right) t\right],(1
\end{gathered}
$$

where $G$ is the Newtonian constant of gravitation, $\varepsilon_{\mathrm{T}}=$ $\left(R_{1} / A\right)^{3}\left(m_{2} / m_{1}\right)$ is the ratio of the tidal force to the gravity at the star's equator, $\boldsymbol{r}=(r, \theta, \phi)$ is a system of spherical coordinates with respect to an orthogonal frame of reference that is corotating with the star, $\tau$ is a time of periastron passage, the $Y_{\ell}^{m}(\theta, \phi)$ are unnormalised spherical harmonics, and the $c_{\ell, m, k}$ are Fourier coefficients depending on the orbital eccentricity (for a definition see Smeyers et al. 1998).

The rate of secular apsidal motion due to the tidal distortion of the components of a close binary is usually derived under the assumption that the orbital period is long in comparison to the periods of the free oscillation modes of the component stars (Cowling 1938; Sterne 1939). The resulting formula takes the form

$\dot{\omega}_{\text {tidal }}=\left(\frac{R_{1}}{A}\right)^{5} \frac{m_{2}}{m_{1}} \frac{2 \pi}{P} k_{2} 15 g(e)$.

In binaries with shorter orbital periods, deviations from the classical formula arise due to the effects of the stellar compressibility and due to the occurrence of resonances between dynamic tides and free oscillation modes (Smeyers \& Willems 2001; Willems \& Claret 2002). When the expansion of the tide-generating potential is restricted to the dominant $\ell=2$ terms, the formula for the rate of secular apsidal motion taking into account the effects of stellar compressibility and resonances is given by

$$
\begin{aligned}
\dot{\omega}_{\mathrm{dyn}}= & \left(\frac{R_{1}}{A}\right)^{5} \frac{m_{2}}{m_{1}} \frac{2 \pi}{P}\left[2 k_{2} G_{2,0,0}+4 \sum_{k=1}^{\infty}\left(F_{2,0, k} G_{2,0, k}\right.\right. \\
& \left.\left.+F_{2,2, k} G_{2,2, k}+F_{2,-2, k} G_{2,-2, k}\right)\right] .
\end{aligned}
$$

The equation depends on the orbital eccentricity through the factors $G_{2, m, k}$ and on the response of the star to the tidal forcing through the coefficients $F_{2, m, k}$. For details on the quantities $G_{2, m, k}$ and $F_{2, m, k}$, we refer to Smeyers \& Willems (2001) and Willems \& Claret (2002).

In the context of this investigation, it is furthermore convenient to rewrite Eq. (14) in the form

$\dot{\omega}_{\mathrm{dyn}}=\left(\frac{R_{1}}{A}\right)^{5} \frac{m_{2}}{m_{1}} \frac{2 \pi}{P} k_{2, \mathrm{dyn}} 15 g(e)$

with

$$
\begin{aligned}
k_{2, \mathrm{dyn}}= & \frac{1}{15 g(e)}\left[2 k_{2} G_{2,0,0}+4 \sum_{k=1}^{\infty}\left(F_{2,0, k} G_{2,0, k}\right.\right. \\
& \left.\left.+F_{2,2, k} G_{2,2, k}+F_{2,-2, k} G_{2,-2, k}\right)\right] .
\end{aligned}
$$

Since the values of the function $15 g(e)$ are close to those of the function

$2 G_{2,0,0}+4 \sum_{k=1}^{\infty}\left(G_{2,0, k}+G_{2,2, k}+G_{2,-2, k}\right)$

up to high orbital eccentricities (Smeyers \& Willems 2001), the constant $k_{2 \text {,dyn }}$ can be considered as a weighted mean of the coefficients $F_{2, m, k}$ with the weights depending on the orbital eccentricity.

In order to determine rate of secular apsidal motion within the framework of the theory of dynamic tides, the contribution of each term in Eq. (14) is determined by numerical integration of the system of differential equations governing forced isentropic oscillations of a spherically symmetric star. As the periods of the eclipsing binaries listed in Table 1 are known with high accuracy, we here calculated the contribution of the tidal distortion to the apsidal-motion rate as a function of $\Omega_{1} / \Omega_{\mathrm{P}}$, where $\Omega_{\mathrm{P}}$ is the orbital angular velocity at the periastron. The resulting relative deviations

$$
\Delta_{\mathrm{dyn}} \equiv \frac{\dot{\omega}_{\mathrm{tidal}}-\dot{\omega}_{\mathrm{dyn}}}{\dot{\omega}_{\mathrm{dyn}}}=\frac{k_{2}-k_{2, \mathrm{dyn}}}{k_{2, \mathrm{dyn}}}
$$

between the rate of secular apsidal motion given by the classical formula and the corresponding rate determined within the framework of the theory of dynamic tides are displayed by the solid lines in Fig. 4 for $\Omega_{1}$ ranging from $0.01 \Omega_{\mathrm{P}}$ to $3 \Omega_{\mathrm{P}}$. The dotted vertical lines in the figures correspond to the observed values of $\Omega_{1} / \Omega_{\mathrm{P}}$ resulting from Table 1.

The relative differences $\Delta_{\text {dyn }}$ can be positive or negative, depending on the level of synchronization at the periastron. Such a tendency was already detected in the paper by Willems \& Claret (2002) under the hypothesis that the systems rotated slowly in comparison to their orbital motion. In the cases of VV Pyx, EK Cep, and GG Ori, the relative deviations are solely due to the effects of the stellar compressibility. In all other cases, additional deviations may arise, in particular for higher rotational angular velocities, due to the occurrence of resonances when the forcing frequencies of the lower-order harmonics in the 

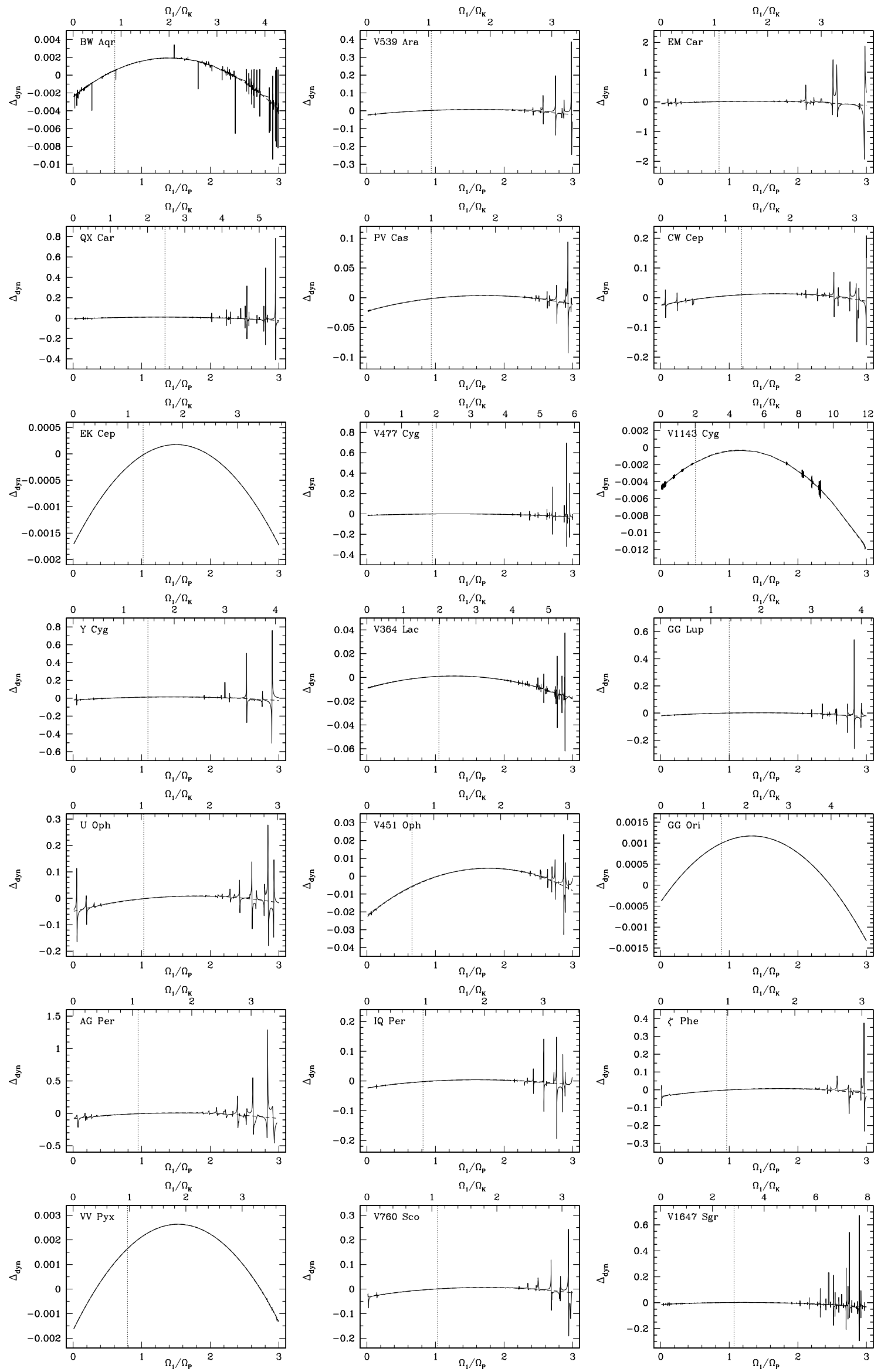

Fig. 4. The deviations from the classical apsidal-motion constant caused by dynamic tides for the DLEBS listed in Table 1. 
Table 2. Coefficients $d_{i}$ of the polynomials used to fit the relative differences $\Delta_{\text {dyn }}$ for the systems listed in Table 1.

\begin{tabular}{llll}
\hline \hline Name & $d_{0}$ & $d_{1}$ & $d_{2}$ \\
\hline BW Aqr & -0.002439 & 0.006274 & -0.002252 \\
V539 Ara & -0.02379 & 0.04086 & -0.01340 \\
EM Car & -0.06835 & 0.1370 & -0.05129 \\
QX Car & -0.008538 & 0.02813 & -0.01101 \\
PV Cas & -0.02230 & 0.03038 & -0.008872 \\
CW Cep & -0.02600 & 0.04736 & -0.01419 \\
EK Cep & -0.001729 & 0.002540 & -0.0008462 \\
V477 Cyg & -0.01455 & 0.02349 & -0.009402 \\
V1143 Cyg & -0.004806 & 0.007856 & -0.003404 \\
Y Cyg & -0.02052 & 0.04837 & -0.01664 \\
V364 Lac & -0.009066 & 0.01626 & -0.006418 \\
GG Lup & -0.01963 & 0.03153 & -0.01128 \\
U Oph & -0.04964 & 0.06527 & -0.01810 \\
V451 Oph & -0.02204 & 0.02997 & -0.008438 \\
GG Ori & -0.0004012 & 0.002373 & -0.0008941 \\
AG Per & -0.08293 & 0.1206 & -0.03986 \\
IQ Per & -0.02310 & 0.03288 & -0.009911 \\
$\zeta$ Phe & -0.03637 & 0.05202 & -0.01555 \\
VV Pyx & -0.001662 & 0.005618 & -0.001835 \\
V760 Sco & -0.03150 & 0.04466 & -0.01311 \\
V1647 Sgr & -0.01351 & 0.02503 & -0.01037 \\
\hline
\end{tabular}

expansion of the tidal potential come into the range of the frequencies of the lower-order gravity modes of the star.

The main trend of the relative differences $\Delta_{\text {dyn }}$ in the absence of resonances can be approximated by a seconddegree polynomial of the form

$\Delta_{\mathrm{dyn}}^{\mathrm{fit}}=d_{0}+d_{1}\left(\Omega / \Omega_{\mathrm{P}}\right)+d_{2}\left(\Omega / \Omega_{\mathrm{P}}\right)^{2}$.

For the determination of the coefficients $d_{i}$, we used a least-squares procedure and fitted the above equation to the relative differences $\Delta_{\text {dyn }}$ displayed in Fig. 4 . The resulting coefficients $d_{i}$ are listed in Table 2. The fits are shown as dashed lines in the various panels of Fig. 4.

\subsection{Comparison with observations}

Once our systems have passed the various tests concerning masses, radii, ages and effective temperatures we are able to proceed to the comparison between the observed values of $k_{2}$ and the values predicted by our stellar evolution code after averaging as in Eq. (11). It is however worth mentioning first that an additional time dependence in the position of the apses can be induced by, e.g., the presence of a third body, the effect of the circumstellar material, and the inclination of the rotational axes with respect to the orbital angular momentum axis. There is no evidence for the detection of any of these phenomena in our sample, but it is also true that only few attempts were made to detect them. Therefore, we will only consider the effects of the relativistic contribution, dynamic tides, stellar rotation, mass loss, and moderate core overshooting on the classical contribution. The correction introduced by rotating models is defined as $\Delta \log k_{2} \equiv \log k_{2 \text { stan }}-\lambda$, where

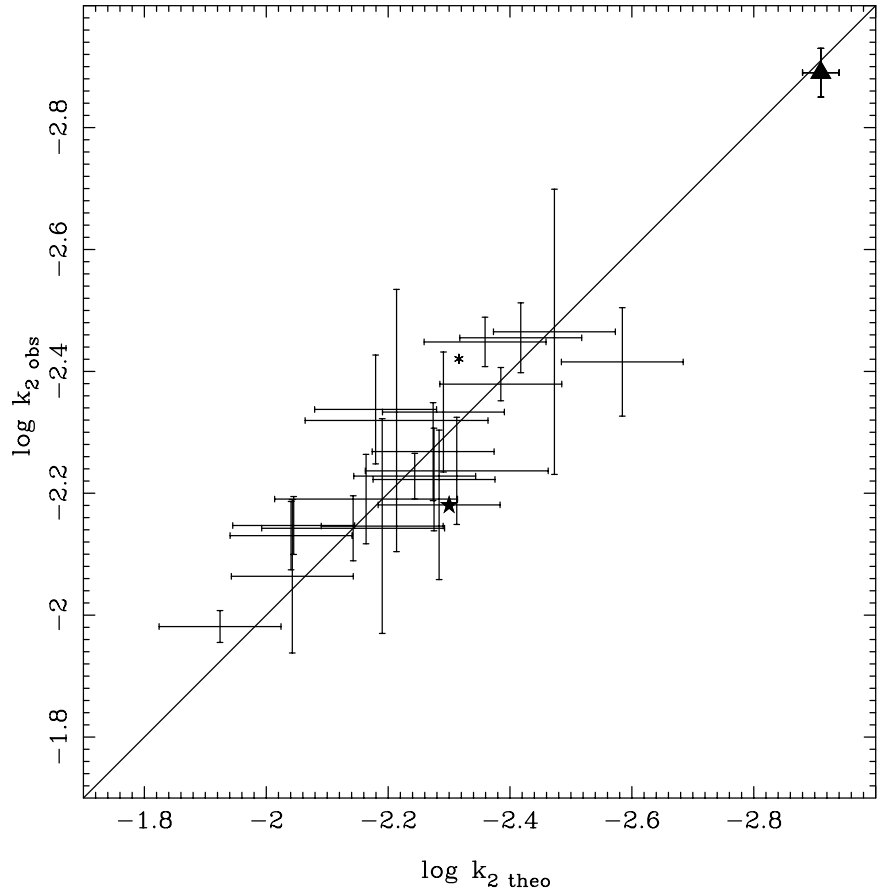

Fig. 5. Comparison between the theoretical predictions for the apsidal-motion constants and the observed counterparts. The asterisk, the star and the triangle represent GG Ori, IT Cas and V380 Cyg, respectively.

$\lambda=2 V^{2} /(3 g R)$ and $V$ is the rotational velocity (Claret 1999).

The resulting comparison between observed and theoretical apsidal-motion constants is shown in Fig. 5. The mentioned corrections used to calculate the theoretical apsidal-motion constants are listed in Table 3. The comparison can be considered as good. The error bars for the system GG Ori are not represented due to the large associated error in the observed apsidal-motion rate. This binary is denoted by an asterisk. The largest theoretical error bars correspond to the four systems mentioned in Sect. 4.1. The similarity between Figs. 5 and 12 by Claret \& Giménez (1993) is clear for values of $\log k_{2}$ between -1.7 and -2.7 but the comparison shows clear differences too. As pointed out previously, the present sample is almost $50 \%$ larger than before (considering only the DLEBS with accurate astrophysical parameters). Of course, this observational improvement provides more confidence and makes the apsidal-motion test a more robust tool to test stellar models. In fact, we have used classical as well as relativistic systems while in the past the latter were investigated separately in order to avoid misinterpretations. The system IT Cas was also included in Figs. 5 and 6 and is denoted by a star. However, we did not consider this system in our present investigation since there seems to be a discrepancy in its observed apsidal-motion rate (Lacy et al. 1997; Kozyreva \& Zakharov 2001). 
Table 3. The classical and general relativistic contributions to apsidal-motion rates, and the corrections to the classical contribution due to rotation and dynamic tides for the systems listed in Table 1.

\begin{tabular}{llllc}
\hline \hline Name & $\begin{array}{l}\text { Classical } \\
\text { deg/cycle }\end{array}$ & $\begin{array}{l}\text { Relativistic } \\
\text { deg/cycle }\end{array}$ & $\begin{array}{l}\lambda \\
\text { in log } k_{2}\end{array}$ & $\Delta_{\text {dyn }}$ \\
\hline BW Aqr & 0.00058 & 0.00032 & 0.00072 & 0.00054 \\
V539 Ara & 0.01971 & 0.00129 & 0.01195 & 0.0027 \\
EM Car & 0.07796 & 0.00304 & 0.02876 & 0.011 \\
QX Car & 0.01075 & 0.00147 & 0.02189 & 0.0094 \\
PV Cas & 0.01742 & 0.00118 & 0.01198 & -0.0016 \\
CW Cep & 0.05660 & 0.00242 & 0.02693 & 0.010 \\
EK Cep & 0.00057 & 0.00044 & 0.00093 & -0.000019 \\
V477 Cyg & 0.00588 & 0.00073 & 0.01101 & -0.00069 \\
V1143 Cyg & 0.00035 & 0.00039 & 0.00195 & -0.0017 \\
Y Cyg & 0.05896 & 0.00284 & 0.02322 & 0.012 \\
V364 Lac & 0.00214 & 0.00044 & 0.00713 & 0.00093 \\
GG Lup & 0.01680 & 0.00130 & 0.01408 & 0.00061 \\
U Oph & 0.07613 & 0.00177 & 0.02288 & -0.0016 \\
V451 Oph & 0.01084 & 0.00096 & 0.00484 & -0.0060 \\
GG Ori & 0.00016 & 0.00045 & 0.00110 & 0.0010 \\
AG Per & 0.02479 & 0.00161 & 0.01465 & -0.0044 \\
IQ Per & 0.01326 & 0.00114 & 0.00989 & -0.0028 \\
S Phe & 0.03596 & 0.00134 & 0.01753 & -0.00072 \\
VV Pyx & 0.00090 & 0.00052 & 0.00191 & 0.0016 \\
V760 Sco & 0.04210 & 0.00170 & 0.01769 & 0.00059 \\
V1647 Sgr & 0.00469 & 0.00077 & 0.01694 & 0.0014 \\
\hline
\end{tabular}

\subsection{Correlation with the evolutionary stage}

One of the main results from Claret \& Giménez (1993) was that the discrepancies between the observed and theoretical $k_{2}$ seemed to be correlated with the evolutionary status of the system (Fig. 8 of that paper). However, most of the points which defined such a trend were due to systems with lower accuracy ( $\alpha$ Vir, V1765 Cyg and V380 Cyg). In order to test if this tendency still exists in our present sample, an $\mathrm{O}-\mathrm{C}$ diagram $\left(\delta \equiv \log k_{2 \mathrm{obs}}-\log k_{2 \text { theo }}\right)$ as a function of the observed surface gravity is shown in Fig. 6 . The new input physics and the severe selection processes to which we have submitted the present sample remove the discrepancies found in the past. A similar tendency can also be deduced from Fig. 8 by Claret \& Giménez if less accurate systems (crosses) are not taken into account. In this case, however, a small residual disagreement is still detected for the systems with accurate absolute dimensions.

The role of the evolved systems shown in the mentioned Fig. 8 will be re-examined in a separate investigation. The absolute dimensions and apsidal-motion rates for $\alpha$ Vir and V1765 Cyg are still not accurate enough to reliably apply the apsidal-motion test (see for example, the light curve of V1765 Cyg in Mayer et al. 1991), and were therefore excluded from the present sample. On the contrary, V380 Cyg was recently revised by Guinan et al. (2000) who derived more precise stellar and orbital parameters. The authors also achieved a good agreement between the observed and theoretical apsidal-motion rates by adopting the results of the same evolution code used

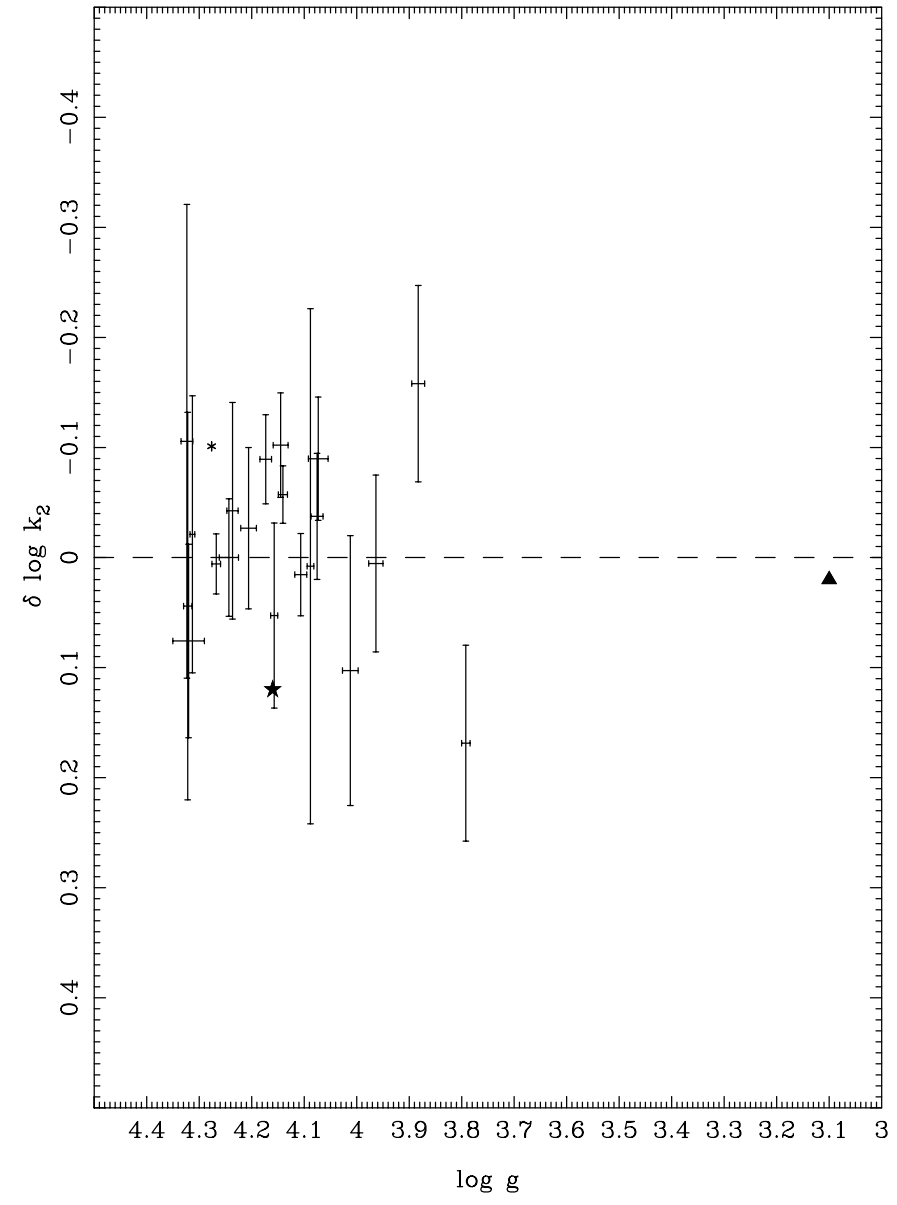

Fig. 6. Differences between the observed and predicted apsidal-motion constants as a function of the evolutionary status. The asterisk, the star and the triangle represent GG Ori, IT Cas and V380 Cyg, respectively.

here (see also Claret 2002). The inclusion of this system in our present sample would therefore not change the good agreement found in Figs. 5 and 6.

The case of DI Her is different since the absolute dimensions are well determined but the derivation of $U$ is complicated given the large difference between the two time scales involved: on the one hand there is the long apsidal-motion period $U$ ( $\approx 46000$ years), and on the other hand there is the time interval between observations of minimum light (only a few decades). The different techniques and the different instruments used in this interval do not provide similar accuracies. Morevover, the position of the periastron seems not to be favorable for accurate observations. For a more extensive discussion, see Claret (1998).

\section{Remaining uncertainties and future improvements}

\subsection{Observational constraints}

The agreement between theory and observations shown in Fig. 5 and the apparent absence of a systematic disagreement correlated with the evolutionary status of the 
systems (Fig. 6) only permit a moderate dose of optimism though. Apart from the determination of the absolute dimensions, the observation of the apsidal-motion rate itself also constitutes a critical element in this context. For those systems for which we expect a large period of apsidal motion, the inherent difficulties to determine it with good accuracy are obvious. If $U$ is large, it is hard to cover the whole period. For many cases the observations therefore cover only a few per cent of $U$. Moreover, the length of the period $U$ often means that different techniques and instruments were used and joining such information in a coherent and consistent way is not straightforward. In more complicated cases, visual and photographic observations of times of minimum light were even discarded. This blurring may have consequences that are hard to control and the results should therefore be taken with caution.

The position of the longitude of the periastron $\omega$ may also limit the quality of the data: since the apsidal-motion rate is a derivative, the position where it is observed is critical. An attempt to obtain $\dot{\omega}$ near its maximum or minimum may be affected by serious errors. It is interesting to point out that in some of the puzzling binaries, mainly among the relativistic ones, all these problematic circumstances are present (Claret 1997). Another important point which should be considered by the observers is the Rossiter effect connected with the orientation of the rotation axis. If accurate observations are performed during the eclipses, a substantial information on the orientation of the rotation axis can be inferred from the shifts in the center of the lines. As known, depending on the orientation of the rotation axis with respect to the orbital angular momentum, there will be a correction of the rotational contribution to the total apsidal-motion rate. This was described in some detail by Kopal (1978) and later by Sakura (1984).

\subsection{Stellar rotation}

The results displayed in Fig. 5 were obtained using directly the observed rotational velocities. However, we also performed some additional numerical experiments concerning the rotational contribution to the apsidal-motion rate. Instead of using the observed values of $V_{1}$ and $V_{2}$, we recomputed the theoretical and observed $k_{2}$ under the assumption that the systems were synchronized at the periastron. Although rough, this may allow us to evaluate, at least partially, the errors introduced by inaccuracies in the rotational velocities. The mean error in $\log k_{2}$ due to the change of the input rotational velocities is of the order of 0.01 . Of course, the precise magnitude of the deviation depends on the relative contribution of rotation with respect to tides. The hypothesis of synchronism at the periastron is ambiguous in the tidal evolution scenario because it implies that both components are pseudo-synchronized simultaneously, which is not always observed. In a first approximation, this should occur only for systems which have a mass ratio around 1 and for which both components are still on the Main Sequence. Furthermore, the question if these velocities are representative for the interior of the stars also forms an additional source of uncertainties.

\subsection{Physics of the interior and atmosphere models}

In order to be realistic, we should keep in mind some of the problems that are still present in the comparison between theoretical stellar models and observations of DLEBS. Only simultaneous observational and theoretical efforts will make a critical test of the mass distribution in stars possible. Therefore, stellar models must be deeply improved. Several physical ingredients influence the theoretical values of $k_{2}$. Opacities, in particular, are often used to justify disagreements and in fact were partially responsible for sensible improvements during the past decade (see Fig. 2 by Claret \& Giménez 1992). The actual generation of OPAL tables provides a valuable tool for stellar modelling but surely, in the future, new and even more accurate opacities will be available. We estimate the uncertainties produced by the opacities to be around \pm 0.04 but they can increase up to \pm 0.1 for more massive and more evolved stellar models.

The implementation of more elaborate atmospheric models coupled with the envelope may have interesting consequences not only for the absolute dimensions but perhaps also for the mass distribution, mainly in the upper layers of the models.

Another methodologic improvement is to compute the stellar models for the precise observed masses. This avoids interpolation problems often appearing in more evolved systems which are in rapid phases of evolution. Although largely known, the rate of stellar evolution is not constant in time and mass: the observation and interpretation of data from stars positioned in different places of the HR Diagram do not necessarily provide us with the same degree of confidence.

Mass-loss rates (see the recent work by Vink et al. 2001) and rotation finally are phenomena which, a priori, produce more centrally condensed models. The correction we have introduced by rotation is only a first approximation to the problem since the adopted redistribution laws of angular momentum did not take into account rotationally-induced instabilities. The role of the induced mixing (the evolution of the angular velocity is coupled with the associated diffusion equation) is now being explored (Talon et al. 1997). It is important to stress that rotation does not only influence the theoretical computation of $k_{2}$ due to the shifts in luminosity and effective temperature, but that the quoted mixing process may also affect the mass distribution. Its influence on the apsidalmotion constant is currently under investigation.

\subsection{Convection}

The theories of the transport of energy in stars by convection are also still a source of uncertainties for stellar 
modelling. The classical mixing-length theory still survives in spite of the efforts of several theoreticians (e.g. Canuto 1999) to provide a better approximation. Concerning the mass concentration only, core convection is more important than convection in the external layers, but the latter determines the comparison with the absolute dimensions for less massive or evolved stars. On the other hand, if core overshooting is considered, more fuel is available for nuclear burning and, as a consequence, the main-sequence life time is increased. The enlargement of the traditional convective core size, obtained trough the Schwarzschild criterion, seems to be necessary, for example to provide isochrones which can fit several stellar cluster data (see Maeder \& Meynet 1989; Chiosi \& Maeder 1986). Binary star data seem to indicate the need of this extra extension with respect to the absolute dimensions (e.g. Clausen 1991; Andersen 1991; Claret \& Giménez 1993; Ribas 1996) as well as concerning the mass concentration (Claret \& Giménez 1991). In the latter paper, the influence of the amount of overshooting on the apsidal-motion constants was investigated, for the first time, by varying the parameter $\alpha_{\text {ov }}$ from 0 (no overshooting) up to 0.6. Two of the most conflictive binaries as discussed by Claret \& Giménez (1993), namely V380 Cyg and $\alpha$ Vir, were satisfactorily fitted only for $\alpha_{\mathrm{ov}} \approx 0.60$. At that time, this was considered an excessive value. The system V380 Cyg is currently being revised in the light of the recent improvement in the determination of its absolute dimensions (Claret 2002).

Acknowledgements. The Spanish DGESIC (project PB980499) is acknowledged by the support received. BW acknowledges the support of the British Particle Physics and Astronomy Research Council (PPARC). This research has made use of the Simbad database, operated at CDS, Strasbourg, France.

\section{References}

Alexander, D. R., \& Ferguson, J. W. 1994, ApJ, 437, 879

Andersen, J. 1983, A\&A,

Andersen, J. 1991, A\&AR, 3, 91

Andersen, J., \& Clausen, J. V. 1989, A\&A, 213, 183

Andersen, J., Clausen, J. V., Gustafsson, B., Nordström, B., \& VandenBerg, D. A. 1988, A\&A, 196, 128

Andersen, J., \& Giménez, A. 1985, A\&A, 145, 206

Andersen, J., Clausen, J. V., \& Giménez, A. 1993, A\&A, 277, 487

Andersen, J., Clausen, J. V., Nordström, B., \& Reipurth, B. 1983, A\&A, 121, 271

Andersen, J., Clausen, J. V., \& Nordström, B. 1984, A\&A, 134, 147

Andersen, J., Nordström, B., \& Popper, D. M. 1985, A\&A, 151,228

Andersen, J., García, J. M., Giménez, A., \& Nordström, B. 1987, A\&A, 174, 107

Audouze J. 1987, in Observational Cosmology, IAU Symp. 124, ed. A. Hewit et al. (Reidel Publishers), 89

Barembaum, M. J., \& Etzel, P. B. 1995, AJ, 109, 2680

Böhm-Vitense, E. 1958, Ze. Astrophys., 46, 108

Burns, J. F., Guinan, E. F., \& Marshall, J. J. 1996, IBVS, 4363

Canuto, V. M. 1999, ApJ, 518, L119
Caton, D. B., \& Burns, W. C. 1993, IBVS, No. 3900

Caton, D. B., Hawkins, R. L., \& Burns, W. C. 1989, IBVS, No. 3408

Chiosi, C., \& Maeder, A. 1986, ARA\&A, 24, 329

Claret, A. 1995, A\&AS, 109, 441

Claret, A. 1997, A\&A, 327, 11

Claret, A. 1998, A\&A, 330, 533

Claret, A. 1999, A\&A, 350, 56

Claret, A. 2002, in preparation

Claret, A., \& Giménez, A. 1989, A\&AS, 81, 1

Claret, A., \& Giménez, A. 1991, A\&A, 244, 319

Claret, A., \& Giménez, A. 1992, A\&AS, 96, 255

Claret, A., \& Giménez, A. 1993, A\&A, 277, 487

Claret, A., Giménez, A., \& Martín, E. L. 1995, A\&A, 302, 741

Clausen, J. V., Gyldenkerne, K., \& Gronbech, B. 1977, A\&A, 58,131

Clausen, J. V. 1991, A\&A, 246, 397

Clausen, J. V. 1996, A\&A, 308, 151

Clausen, J. V., Gyldenkerne, K., \& Gronbech, B. 1977, A\&A, 58,121

Clausen, J. V., \& Giménez, A. 1991, A\&A, 241, 98

Clausen, J. V., \& Giménez, A. 1994, A\&A, 291, 795

Clausen, J. V., Giménez, A., \& Scarfe, C. 1986, A\&A, 167, 287

Clausen, J. V., Baraffe, I., Claret, A., \& VandenBerg, D. A. 1999, in Theory and tests of convection in stellar structure, ASP Conf. Ser., 173, ed. A. Giménez, E. F. Guinan, \& B. Montesinos, 265

Clausen, J. V., Helt, B. E., \& Olsen, E. H. 2001, A\&A, 374, 980

Cowling, T. G. 1938, MNRAS, 98, 734

Diethelm, R. 1992, Bull. BBSAG, 89

Diethelm, R. 1993, IBVS, No. 3903

Giménez, A., \& Margrave, T. E. 1985, AJ, 90, 358

Giménez, A., Clausen, J. V., \& Jensen, K. S. 1986, A\&A, 159, 157

Giménez, A., \& Quintana, J. M. 1992, A\&A, 260, 227

Giménez, A., \& Clausen, J. V. 1994, A\&A, 291, 795

Gronbech, B., Andersen, J., Clausen, J. V., Helt, B. E., \& Jensen, K. S. 1987, A\&AS, 68, 323

Guinan, E., Ribas, I., Fitzpatrick, E. L., et al. 2000, ApJ, 544, 409

Hill, G., \& Ebbighausen, E. G. 1984, AJ, 89, 1256

Hill, G., \& Holmgren, D. E. 1995, A\&A, 297, 127

Holmgren, D. E., Hill, G., \& Scarfe, C. D. 1995, Observatory (The), 115, 188

Iglesias, C. A., \& Rogers, F. J. 1996, ApJ, 459, L35

Imbert, M. 1987, A\&AS, 69, 397

Kämper, B. C. 1986, Ap\&SS, 120, 167

Khaliullin, Kh. F. 1983, Sov. Astron., 27, 43

Khaliullin, Kh. F. 1983, Astron. Tsirk., No. 1262

Khaliullin, Kh. F., \& Kozureva, V. S. 1986, Ap\&SS, 120, 9

Kozyreva, V. S., \& Zakharov, A. I. 2001, Astron. Lett., 27, 712

Kippenhahn, R., \& Thomas, R. C. 1970, in Stellar Rotation, ed. A. Slettebak (D. Reidel, Publ. Co., Dordrecht, Holland), 20

Kurucz R. L. 1994, private communication

Kopal, Z. 1959, Close binary systems (Chapman and Hall, London)

Kopal, Z. 1978, Dynamics of Close Binary Systems (Reidel, Dordrecht, Holland)

Krzesinski, J., Kuczawska, E., \& Pajdosz, G. 1990, IBVS, No. 3495

Lacy, C. H., \& Frueh, M. L. 1985, ApJ, 295, 569 
Lacy, C. H., Torres, G., Latham, D. W., Zakirov, M. M., \& Arzumanyants, G. C. 1997, AJ, 114, 12067

Torres, G., Lacy, C. H., Claret, A., \& Sabby, J. A. 2000, AJ, 120,3226

Levi-Civita, T. 1937, Amer. J. Math., 59, 225

Linell, A. P., Hubeny, I., \& Lacy, C. H. S. 1996, ApJ, 459, 721

Maeder, A.1983, Proceedings of ESO Workshop on primordial helium, ed. P. S. Saver, D. Kunth, \& Kjär, 89

Maeder, A., \& Meynet, G. 1989, A\&A, 210, 155

Maloney, F. P., Guinan, E. F., \& Mukherjee, J. 1991, AJ, 102, 256

Martín, E. L., \& Rebolo, R. 1993, A\&A, 274, 274

Mayer, P., Hadrava, P., Harmanec, P., \& Chochol, B. 1991, BAICz, 42, 230

Moffat, J. W. 1984, ApJ, 287, L77

Moffat, J. W. 1989, Phys. Rev. D, 39, 474

Pajdosz, G. 1993, private communication to A. Giménez Papaloizou, J., \& Pringle, J. E. 1980, MNRAS, 193, 603

Polfliet, R., \& Smeyers, P. 1990, A\&A, 237, 110

Popper, D. M. 1987, AJ, 93, 672

Popper, D. M. 1987, ApJ, 313, L81

Popper, D. M., \& Hill, G. 1991, AJ, 101, 600

Popper, D. M., \& Hill, G. 1991, AJ, 102, 1156

Quataert, E. J., Kumar, P., \& Ao, C. O. 1996, ApJ, 463, 284

Ribas, I., Jordi, C., Torra, J., \& Giménez, A. 2000, MNRAS, 313, 99
Ribas, I. 1996, Ph.D. Thesis, University of Barcelona

Russell, H. N. 1928, MNRAS, 88, 641

Sakura, N. I. 1984, Sov. Astr. Lett., 11(4), 225

Smeyers, P., \& Willems, B. 2001, A\&A, 373, 173

Smeyers, P., Willems, B., \& Van Hoolst, T. 1998, A\&A, 335, 622

Simon, K. P., Sturm, E., \& Fiedler, A. 1994, A\&A, 292, 507

Sterne, T. E. 1939, MNRAS, 99, 451

Stothers, R. 1974, ApJ, 194, 651

Talon, S., Zahn, J-P., Maeder, A., \& Meynet, A. 1997, A\&A, 322,209

Tomkin, J. 1983, ApJ, 271, 717

Torres, G., Lacy, Claud, H. S., et al. 1999, AJ, 118, 1831

Torres, G., Stefanik, R. P., Andersen, J., et al. 1997, AJ, 114, 2764

Torres, G., Lacy, C. H., Claret, A., \& Sabby, J. A. 2000, AJ, 120,3226

Vink, J. S., de Koter, A., \& Lamers, H. J. G. L. M. 2001, A\&A, 369,574

Willems, B., \& Claret, A. 2002, A\&A, 382, 1009

Wolf, M. 1995, MNRAS, 277, 95

Wolf, M. 2000, A\&A, 356, 134

Wolf, M., Diethlem, R., \& Hornoch, K. 2001, A\&A, 374, 243

Zahn, J. P. 1970, A\&A, 4, 452 